\title{
EXISTENCE OF SOLUTIONS OF NONLINEAR \\ INTEGRODIFFERENTIAL EQUATIONS WITH NONLOCAL CONDITIONS
}

\author{
A. ANGURAJ, A. R. NAVANEETHAN AND T. S. SUKANYA
}

\begin{abstract}
In this paper we prove the existence of mild and strong solutions of semilinear integrodifferential equations in Banach spaces with nonlocal initial conditions. We prove the existence theorems by using Schaefer's fixed point theorem.
\end{abstract}

\section{Introduction}

In this paper we consider semilinear integrodifferential equations with nonlocal conditions

$$
\begin{aligned}
& y^{\prime}(t)=A(t, y) y+f\left(t, y, \int_{0}^{t} K(t, s) F(s, y(s)) d s\right), \quad t \in J=[0, b], \\
& y(0)+g(y)=y_{0},
\end{aligned}
$$

where $A(t, y): E \rightarrow E, f: J \times E \times E \rightarrow E$ and $K: J \times J \rightarrow R$ are continuous functions, $g: C(J, E) \rightarrow E, y_{0} \in E$ and $E$ is a real Banach space with the norm $\|\cdot\|$. We prove the existence of mild and strong solutions for the above problem by using Schaefer's fixed point theorem.

Nonlinear differential equations with classical initial conditions have been studied by many authors $[1,5]$. The nonlocal conditions which is a generalization of the classical initial condition was considered by Byszewski [4]. Several papers have been devoted to studying the existence of solutions for differential equations with nonlocal conditions $[2,3,4]$. In this paper we investigate the mild and strong solutions of the semilinear integrodifferential equations with nonlocal conditions in Banach spaces.

\section{Preliminaries and Basic Hypotheses}

Let $C(J, E)$ be the Banach space of continuous functions from $J$ into $E$ normed by

$$
\|y\|_{\infty}=\sup \{\|y(t)\| / t \in J\} .
$$

Received and revised May 10, 2004.

Key words and phrases. Existence, mild soultion, strong solution, integrodifferential equations, nonlocal condition, fixed point. 
and let $B(E)$ denote the Banach space of bounded linear operators from $E$ into $E$ with norm

$$
\|N\|_{B(E)}=\sup \{\|N y\| /\|y\|=1\} .
$$

The following lemma and fixed point theorem are useful for proving the existence theorems.

Lemma 1. ([6], p.36) Suppose that $\Phi_{1}, \Phi_{2} \in C(J, R), \Phi_{3} \in L^{1}(J, R), \Phi_{3}(t) \geq 0$ a.e. on $J$ and $\Phi_{1}(t) \leq \Phi_{2}(t)+\int_{0}^{t} \Phi_{3}(s) \Phi_{1}(s) d s$. Then

$$
\Phi_{1}(t) \leq \Phi_{2}(t)+\int_{0}^{t} \Phi_{3}(s) \Phi_{2}(s) \exp \left(\int_{s}^{t} \Phi_{3}(\tau) d \tau\right) d s
$$

Fixed point theorem: [7]

Let $E$ be a Banach space and let $N: E \rightarrow E$ be a continuous compact map. If the set $\Omega=\{y \in E: \lambda y=N(y)$ for some $\lambda>1\}$ is bounded, then $N$ has a fixed point.

Now, let us assume the following hypotheses:

(H1) $A: J \times E \rightarrow B(E)$ is a continuous function such that $\forall r>0, \exists r_{1}=r_{1}(r)>0$ such that $\|v\| \leq r \Rightarrow\|A(t, v)\|_{B(E)} \leq r_{1}, \forall t \in J, \forall v \in E$.

(H2) $f: J \times E \times E \rightarrow E,(t, u, v) \rightarrow f(t, u, v)$ is a continuous function.

(H3) There exists a constant $L>0$ such that $\|g(y)\| \leq L$ for some $y \in E$.

(H4) $K: J \times J \rightarrow R$ is a continuous function such that $\|K(t, s)\| \leq L_{1}$ for some constant $L_{1}$ and for some $t, s \in J$.

(H5) $F: J \times E \rightarrow E$ is a continuous function.

(H6) $\left\|F(t, x)_{1}\right\| \leq q(t) H(\|x(t)\|)$ for almost all $t \in J$ and all $x \in E$, where $q \in$ $L^{1}\left(J, R_{+}\right)$and $H: R_{+} \rightarrow(0, \infty)$ is continuous and non-decreasing.

(H7) $\|f(t, y, z)\| \leq p(t) \psi(\|y\|+\|z\|)$ for almost all $t \in J$ and all $y, z \in E$, where $p \in L^{1}\left(J, R_{+}\right)$and $\psi: R_{+} \rightarrow(0, \infty)$ is continuous and non-decreasing with

$$
M \int_{0}^{b} p(s) d s<\int_{c}^{\infty} \frac{d u}{\psi(u)},
$$

where $c=M\left\|y_{0}\right\|+M L$ and $M=\sup \left\{\left\|U_{y}(t, s)\right\|_{B(E)} /(t, s) \in J \times J\right\}$.

(H8) For each bounded $B \subset C(J, E), y \in B$ and $t \in J$ the set $\left\{U_{y}(t, 0) y_{0}-U_{y}(t, 0)\right.$ $\left.g(y)+\int_{0}^{t} U_{y}(t, s) f(s, y(s), z(s)) d s\right\}$, where $z(s)=\int_{0}^{t} K(t, s) F(s, y(s)) d s$, is relatively compact.

(H9) $\int_{0}^{t} M_{1}(s) d s<\int_{c}^{\infty} \frac{d s}{M \psi(s)+H(s)}$, where $M_{1}(t)=\max \left\{p(t), L_{1} q(t)\right\}$.

Remark. From (H1) for any fixed $u \in C(J, E)$ there exists a unique function $U_{n}$ : $J \times J \rightarrow B(E)$ defined and continuous on $J \times J$ such that

$$
U_{n}(t, s)=I+\int_{S}^{t} A_{u}(w) U_{u}(w, s) d w
$$

where $I$ stands for the identity operator on $E$ and $A_{u}(t)=A(t, u(t)) . U_{n}(t, s)$ is the evolution operator of $A$. 
Definition 1. A continuous solution $y(t)$ of the integral equation

$$
y(t)=U_{y}(t, 0) y_{0}-U_{y}(t, 0) g(y)+\int_{0}^{t} U_{y}(t, s) f(s, y(s), z(s)) d s
$$

is called a mild solution of (1).

Definition 2. A function $y$ is said to be a strong solution of (1) on $J$ if, $y$ is differentiable almost everywhere on $J$,

$$
\begin{gathered}
\frac{d y}{d t} \in\left(L^{1}[0, b], E\right), \\
y^{\prime}=A(t, y) y+f(t, y, z) \text { a.e. on } J \text { and } y(0)+g(y)=y_{0} .
\end{gathered}
$$

\section{Existence Theorems}

\subsection{Mild solutions}

Theorem 1. Let $g: C(J, E) \rightarrow E$ be a continuous function. Assume that hypotheses (H1)-(H8) are satisfied. Then problem (1) has at least one mild solution on $J$.

Proof. Consider the map $N: C(J, E) \rightarrow C(J, E)$ defined by

$$
(N y)(t)=U_{y}(t, 0) y_{0}-U_{y}(t, 0) g(y)+\int_{0}^{t} U_{y}(t, s) f(s, y(s), z(s)) d s,
$$

where $t \in J$.

Step 1: $U_{u}(t, s)$ is continuous with respect to $u$; that is,

$$
\begin{aligned}
& \left\|u_{n}-u^{*}\right\|_{\infty} \rightarrow 0 \Rightarrow \\
& \left\|U_{u_{n}}-U_{u^{*}}\right\|_{\infty}=\sup _{(t, s) \in J \times J}\left\|U_{u_{n}}(t, s)-U_{u^{*}}(t, s)\right\|_{B(E)} \rightarrow 0, \text { as } n \rightarrow \infty .
\end{aligned}
$$

Let $\left\|u_{n}-u^{*}\right\|_{\infty} \rightarrow 0$. Then there exists $r>0$ such that $\left\|u_{n}\right\|_{\infty},\left\|u^{*}\right\|_{\infty} \leq r$. If $s \leq t$, then

$$
\begin{aligned}
\left\|U_{u_{n}}-U_{u^{*}}\right\|_{\infty}= & \sup _{(t, s) \in J \times J}\left\|U_{u_{n}}(t, s)-U_{u^{*}}(t, s)\right\|_{B(E)} \\
\leq & \sup _{(t, s) \in J \times J} \int_{s}^{t}\left\|U_{u_{n}}(w, s)\right\|_{B(E)}\left\|\left[A_{u_{n}}(w)-A_{u^{*}}(w)\right]\right\|_{B(E)} d w \\
& +\sup _{(t, s) \in J \times J} \int_{s}^{t}\left\|A_{u^{*}}(w)\right\|_{B(E)}\left\|\left[U_{u_{n}}(w, s)-U_{u^{*}}(w, s)\right]\right\|_{B(E)} d w \\
\leq & \int_{s}^{t} M\left\|\left[A_{u_{n}}(w)-A_{u^{*}}(w)\right]\right\|_{B(E)} d w \\
& +\int_{s}^{t}\left\|A_{u^{*}}\right\|_{B(E)}\left\|\left[U_{u_{n}}(w, s)-U_{u^{*}}(w, s)\right]\right\|_{B(E)} d w .
\end{aligned}
$$


By Lemma 1,

$$
\begin{aligned}
\left\|U_{u_{n}}-U_{u^{*}}\right\|_{\infty} \leq & M \int_{s}^{t}\left\|\left[A_{u_{n}}(w)-A_{u^{*}}(w)\right]\right\|_{B(E)} d w \\
& +M \int_{s}^{t}\left\|A_{u^{*}}(w)\right\|_{B(E)}\left(\int_{s}^{t}\left\|A_{u_{n}}(\tau)-A_{u^{*}}(\tau)\right\|_{B(E)} d \tau\right) \\
& \times\left(\exp \int_{w}^{t}\left\|A_{u^{*}}(z)\right\|_{B(E)} d z\right) d w \\
\leq & M \int_{s}^{t}\left\|A_{u_{n}}-A_{u^{*}}\right\|_{\infty} d w \\
& +M \int_{s}^{t}\left\|A_{u^{*}}\right\|_{\infty}\left(\int_{s}^{t}\left\|A_{u_{n}}-A_{u^{*}}\right\|_{\infty} d \tau\right) \\
& \times \exp \left(\int_{w}^{t}\left\|A_{u^{*}}\right\|_{\infty} d z\right) d w \\
\leq & b M\left\|A_{u_{n}}-A_{u^{*}}\right\|_{\infty}+M \int_{s}^{t}\left\{\left\|A_{u^{*}}\right\|_{\infty} b\left\|_{u_{n}}-A_{u^{*}}\right\|_{\infty}\right. \\
& \left.\times \exp \left(b\left\|A_{u^{*}}\right\|_{\infty}\right)\right\} d w \\
\leq & b M\left\|A_{u_{n}}-A_{u^{*}}\right\|_{\infty}+M b^{2}\left\|A_{u^{*}}\right\|_{\infty}\left\|_{u_{n}}-A_{u^{*}}\right\|_{\infty} \exp \left(b\left\|A_{u^{*}}\right\|_{\infty}\right) \\
\leq & \left\|A_{u_{n}}-A_{u^{*}}\right\|_{\infty} b M\left[1+b r_{1} \exp \left(b r_{1}\right)\right] \quad(\text { by }(\mathrm{H} 1)) .
\end{aligned}
$$

Now, $u \in C(J, E)$ implies $A_{u} \in C(J, B(E))$. Also, $\left\|u_{n}-u^{*}\right\|_{\infty} \rightarrow 0$ implies $\| A_{u_{n}}-$ $A_{u^{*}} \|_{\infty}=\max \left\{\left\|A_{u_{n}}(t)-A_{u^{*}}(t)\right\|_{B(E)} / t \in J\right\} \rightarrow 0$ as $n \rightarrow \infty$. Therefore, $\| U_{u_{n}}-$ $U_{u^{*}} \|_{\infty} \rightarrow 0$ as $n \rightarrow \infty$. Thus $\left\|u_{n}-u^{*}\right\|_{\infty} \rightarrow 0 \Rightarrow\left\|U_{u_{n}}-U_{u^{*}}\right\|_{\infty} \rightarrow 0$ as $n \rightarrow \infty$. Therefore, $U_{n}(t, s)$ is continuous with respect to $u$.

Step 2. $N$ maps bounded sets into relatively compact sets i.e., $N$ is a compact map. Let $B_{r}=\left\{y \in C(J, E) /\|y\|_{\infty} \leq r\right\}$. Clearly $B_{r}$ is a bounded set in $C(J, E)$. For each $t \in J$ we have

$$
(N y)(t)=U_{y}(t, 0) y_{0}-U_{y}(t, 0) g(y)+\int_{0}^{t} U_{y}(t, s) f(s, y(s), z(s)) d s .
$$

Therefore, for each $t \in J$ we have

$$
\begin{aligned}
\|N y\| \leq & \left\|U_{y}(t, 0) y_{0}\right\|+\left\|U_{y}(t, 0) g(y)\right\|+\int_{0}^{t}\left\|U_{y}(t, s) f(s, y(s), z(s))\right\| d s \\
\leq & \left\|U_{y}(t, 0)\right\|_{B(E)}\left\|y_{0}\right\|+\left\|U_{y}(t, 0)\right\|_{B(E)}\|g(y)\| \\
& +\int_{0}^{t}\left\|U_{y}(t, s)\right\|_{B(E)}\|f(s, y(s), z(s))\| d s \\
\leq & M\left\|y_{0}\right\|+M\|g(y)\|+\int_{0}^{t} M\|f(s, y(s), z(s))\| d s \\
\leq & M\left\|y_{0}\right\|+M L+M \int_{0}^{t} p(s) \psi(\|y\|+\|z\|) d s .
\end{aligned}
$$


Consider, $\|y\|_{\infty}=\sup \{\|y(t)\| / t \in J\} \leq r$.

Therefore, $\|y\| \leq \sup _{y \in[0, r]}\{\|y(t)\|\}$,

$$
\begin{aligned}
\|z\| & =\left\|\int_{0}^{t} K(t, s) F(s, y(s)) d s\right\| \\
& \leq L_{1} \int_{0}^{t} q(s) H(\|y(s)\|) d s \\
& \leq L_{1}\left\{\sup _{y \in[0, r]} H(y)\right\} \int_{0}^{t} q(s) d s .
\end{aligned}
$$

Therefore,

$$
\begin{aligned}
& \|y\|+\|z\| \leq \sup _{y \in[0, r]}\|y(t)\|+L_{1}\left\{\sup _{y \in[0, r]} H(y)\right\} \int_{0}^{t} q(s) d s . \\
& \text { Let } \mu(t)=\sup _{y \in[0, r]}\|y(t)\|+L_{1}\left\{\sup _{y \in[0, r]} H(y)\right\} \int_{0}^{t} q(s) d s .
\end{aligned}
$$

Then $\|y\|+\|z\| \leq \mu(t)$.

Since $\psi$ is non-decreasing,

$$
\|y\|+\|z\| \leq \mu(t) \Rightarrow \psi(\|y\|+\|z\|) \leq \psi(\mu(t)) .
$$

Therefore, $\|N y\| \leq M\left\|y_{0}\right\|+M L+M \int_{0}^{t} p(s) \psi(\mu(s)) d s$ and

$$
\|N y\|_{\infty} \leq M\left\|y_{0}\right\|+M L+M \sup _{t \in J}\left(\int_{0}^{t} p(s) d s\right) \max _{y \in B}\left(\sup _{y \in[0, r]} \psi(y)\right) .
$$

Now, let $t_{1}, t_{2} \in J, t_{1}<t_{2}$ and $y \in B_{r}$.

Then

$$
\begin{aligned}
& \left\|(N y)\left(t_{2}\right)-(N y)\left(t_{1}\right)\right\| \\
\leq & \left\|\left\{U_{y}\left(t_{2}, 0\right)-U_{y}\left(t_{1}, 0\right)\right\} y_{0}\right\|+\left\|\left\{U_{y}\left(t_{2}, 0\right)-U_{y}\left(t_{1}, 0\right)\right\} g(y)\right\| \\
& +\left\|\int_{0}^{t_{2}} U_{y}\left(t_{2}, s\right) f(s, y(s), z(s)) d s-\int_{0}^{t_{1}} U_{y}\left(t_{1}, s\right) f(s, y(s), z(s)) d s\right\| \\
\leq & \left\|\left\{U_{y}\left(t_{2}, 0\right)-U_{y}\left(t_{1}, 0\right)\right\} y_{0}\right\|+\left\|\left\{U_{y}\left(t_{2}, 0\right)-U_{y}\left(t_{1}, 0\right)\right\} g(y)\right\| \\
& +\| \int_{0}^{t_{2}} U_{y}\left(t_{2}, s\right) f(s, y(s), z(s)) d s-\int_{0}^{t_{2}} U_{y}\left(t_{1}, s\right) f(s, y(s), z(s)) d s \\
& +\int_{0}^{t_{2}} U_{y}\left(t_{1}, s\right) f(s, y(s), z(s)) d s-\int_{0}^{t_{1}} U_{y}\left(t_{1}, s\right) f(s, y(s), z(s)) d s \| \\
\leq & \left\|U_{y}\left(t_{2}, 0\right)-U_{y}\left(t_{1}, 0\right)\right\|_{B(E)}\left\|y_{0}\right\|+\left\|U_{y}\left(t_{2}, 0\right)-U_{y}\left(t_{1}, 0\right)\right\|_{B(E)}\|g(y)\|
\end{aligned}
$$




$$
\begin{aligned}
& \quad+\left\|\int_{0}^{t_{2}} U_{y}\left(t_{2}, s\right) f(s, y(s), z(s)) d s-\int_{0}^{t_{2}} U_{y}\left(t_{1}, s\right) f(s, y(s), z(s)) d s\right\| \\
& +\left\|\int_{t_{1}}^{t_{2}} U_{y}\left(t_{1}, s\right) f(s, y(s), z(s)) d s\right\| \\
& \leq\left\|U_{y}\left(t_{2}, 0\right)-U_{y}\left(t_{1}, 0\right)\right\|_{B(E)}\left\|y_{0}\right\|+\left\|U_{y}\left(t_{2}, 0\right)-U_{y}\left(t_{1}, 0\right)\right\|_{B(E)} \| L \\
& \quad+\left\|\int_{0}^{t_{2}}\left\{U_{y}\left(t_{2}, s\right)-U_{y}\left(t_{1}, s\right)\right\} p(s) \psi(\|y\|+\|z\|) d s\right\| \\
& \quad+M \int_{t_{1}}^{t_{2}} p(s) \psi(\|y(s)\|+\|z(s)\|) d s .
\end{aligned}
$$

The string of inequalities is bounded by $k\left(t_{2}-t_{1}\right)$ for some $k>0$.

Hence, $N\left(B_{r}\right)$ is an equicontinuous family of functions. Therefore, by Ascoli-Arzela theorem, $N\left(B_{r}\right)$ is relatively compact. Therefore, $N$ maps bounded sets into relatively compact sets, i.e., $N$ is a compact map. We have,

$$
(N y)(t)=U_{y}(t, 0) y_{0}-U_{y}(t, 0) g(y)+\int_{0}^{t} U_{y}(t, s) f(s, y(s), z(s)) d s .
$$

Since $U_{y}, g, f$ are all continuous functions, $N$ is also continuous. Therefore, $N$ is a continuous compact map.

Step 3. The set $\Psi=\{y \in C(J, E) / \lambda y=N(y), \lambda>1\}$ is bounded.

Let $\Omega=\{y \in X / \lambda y=N(y)$ for some $\lambda>1\}$, where $X$ is a Banach space and $N: X \rightarrow X$ is a continuous compact map.

Let $y \in \Omega$.

Then $\lambda y=N(y)$ for some $\lambda>1$.

$$
\begin{aligned}
\lambda y(t) & =(N y)(t) \\
& =U_{y}(t, 0) y_{0}-U_{y}(t, 0) g(y)+\int_{0}^{t} U_{y}(t, s) f(s, y(s), z(s)) d s, \quad t \in J,
\end{aligned}
$$

and therefore

$$
y(t)=\lambda^{-1} U_{y}(t, 0) y_{0}-\lambda^{-1} U_{y}(t, 0) g(y)+\lambda^{-1} \int_{0}^{t} U_{y}(t, s) f(s, y(s), z(s)) d s, \quad t \in J
$$

Now $\lambda>1$ implies $\lambda^{-1} \leq 1$.

Therefore, $\quad y(t) \leq U_{y}(t, 0) y_{0}-U_{y}(t, 0) g(y)+\int_{0}^{t} U_{y}(t, s) f(s, y(s), z(s)) d s, \quad t \in J$.

Hence $\quad\|y(t)\| \leq\left\|U_{y}(t, 0) y_{0}\right\|+\left\|U_{y}(t, 0) g(y)\right\|+\int_{0}^{t}\left\|U_{y}(t, s) f(s, y(s), z(s))\right\| d s$

$$
\leq M\left\|y_{0}\right\|+M L+M \int_{0}^{t} p(s) \psi(\|y(s)\|+\|z(s)\|) d s \text { for } t \in J
$$


Let us take the right-hand side of the above inequality as $v(t)$. Then

$$
v(t)=M\left\|y_{0}\right\|+M L+M \int_{0}^{t} p(s) \psi(\|y(s)\|+\|z(s)\|) d s .
$$

Now, $v(0)=M\left\|y_{0}\right\|+M L$. Thus we have,

$$
v(0)=M\left\|y_{0}\right\|+M L \text { and } \quad\|y(t)\| \leq v(t), \quad t \in J .
$$

Since $\psi$ is non-decreasing,

$$
\|y(t)\| \leq v(t) \Rightarrow \psi(y(t)) \leq \psi(v(t)), \quad t \in J .
$$

Now,

$$
v(t)=M\left\|y_{0}\right\|+M L+M \int_{0}^{t} p(s) \psi(\|y(s)\|+\|z(s)\|) d s .
$$

Therefore,

$$
\begin{aligned}
v^{\prime}(t) & =M p(t) \psi(\|y(t)\|+\|z(t)\|) \\
& \leq M p(t) \psi\left(v(t)+L_{1} \int_{0}^{t} q(s) H(v(s)) d s\right) .
\end{aligned}
$$

Let $u(t)=v(t)+L_{1} \int_{0}^{t} q(s) H(v(s)) d s$.

Then

$$
\begin{aligned}
v(t) & \leq u(t) \text { and } \\
u(0) & =v(0)=M\left\|y_{0}\right\|+M L \\
u(0) & =v^{\prime}(t)+L_{1} q(t) H(v(t)) \\
& \leq M p(t) \psi(u(t))+L_{1} q(t) H(v(t)) \\
& \leq M p(t) \psi(u(t))+L_{1} q(t) H(v(t)) \\
& \leq M_{1}(t)\{M \psi(u(t))+H(u(t))\},
\end{aligned}
$$

where $M_{1}(t)=\max \left\{p(t), L_{1} q(t)\right\}$.

$$
u^{\prime}(t) / M \psi(u(t))+H(u(t)) \leq M_{1}(t) .
$$

Intregrating, we have

$$
\begin{aligned}
\int_{u(0)}^{u(t)} \frac{d s}{M \psi(s)+H(s)} & \leq \int_{0}^{t} M_{1}(s) d s<\int_{0}^{b} M_{1}(s) d s \\
& \leq \int_{u(0)}^{\infty} \frac{d s}{M \psi(s)+H(s)} .
\end{aligned}
$$

This inequality implies that there exists a constant $d$ such that $u(t) \leq d, t \in J$. But $v(t) \leq u(t)$. Therefore, $v(t) \leq d$ for $t \in J$. Also, $\|y(t)\| \leq v(t)$ for $t \in J$. Hence 
$\|y(t)\| \leq d$ for $t \in J$. Therefore, $\|y\|_{\infty} \leq d$, where $d$ depends on the functions $M_{1}, \psi$ and $H$. Now, $y \in \Omega$ and $\|y\|_{\infty} \leq d \Rightarrow \Omega$ is bounded. Set $X=C(J, E)$. Then $\Psi$ is bounded.

Now, $C(J, E)$ is a Banach space and $N: C(J, E) \rightarrow C(J, E)$ is a continuous compact map. Also, the set

$$
\Psi=\{y \in C(J, E): \lambda y=N(y) \text { for } \lambda>1\} \text { is bounded. }
$$

Hence by Schaefer's fixed point theorem, $N$ has a fixed point which is a mild solution of $(1)$.

\subsection{Strong solutions}

Theorem 2. Let $E$ be a reflexive Banach space. Let $g: C(J, E) \rightarrow E$ be a continuous function. Assume that hypotheses (H1)-(H9) are satisfied. Then (1) has a strong solution.

Proof. Since all the assumptions of Theorem 1 are satisfied, (1) has a mild solution. Let $y(t)$ satisfy (1). Let $g_{1}(s)=U_{y}(t, s) y(s)$. This $g_{1}(s)$ is differentiable on $J$,

and

$$
\begin{aligned}
d g_{1} / d s & =\left[(d / d s)\left\{U_{y}(t, s)\right\}\right] y(s)+U_{y}(t, s) y^{\prime}(s) \\
& =U_{y}(t, s) f(s, y(s), z(s)) .
\end{aligned}
$$

Since $f \in L^{1}(J ; E), U_{y}(t, s) f(s, y(s), z(s))$ is integrable. Integrating from 0 to $t$, we get,

$$
\begin{gathered}
\int_{0}^{t} \frac{d g_{1}}{d s} d s=\int_{0}^{t} U_{y}(t, s) f(s, y(s), z(s)) d s, \\
U_{y}(t, t) y(t)-U_{y}(t, 0) y(0)=\int_{0}^{t} U_{y}(t, s) f(s, y(s), z(s)) d s .
\end{gathered}
$$

Thus

$$
y(t)=U_{y}(t, 0) y_{0}-U_{y}(t, 0) g(y)+\int_{0}^{t} U_{y}(t, s) f(s, y(s), z(s)) d s
$$

which is the mild solution of (1).

Now, we will show that this mild solution is a strong solution of (1).

Since $f$ is differentialbe a.e. and $f^{\prime} \in L^{1}(J, E), y$ is differentiable a.e. on $J$ and $y^{\prime} \in L^{1}(J, E)$. Now,

$$
\begin{aligned}
\|y(t+h)-y(t)\| \leq & \left\|\left\{U_{y}(t+h, 0)-U_{y}(t, 0)\right\} y_{0}\right\| \\
& +\left\|\left\{U_{y}(t+h, 0)-U_{y}(t, 0)\right\} g(y)\right\| \\
& +\int_{0}^{t}\left\|\left\{U_{y}(t+h, s)-U_{y}(t, s)\right\} f(s, y(s), z(s))\right\| d s \\
& +\int_{t}^{t+h}\left\|U_{y}(t+h, s) f(s, y(s), z(s))\right\| d s \\
\leq & \left\|U_{y}(t+h, 0)-U_{y}(t, 0)\right\|\left\|y_{0}\right\| \\
& +\left\|U_{y}(t+h, 0)-U_{y}(t, 0)\right\|\|g(y)\|
\end{aligned}
$$




$$
\begin{aligned}
& +\int_{0}^{t}\left\|\left\{U_{y}(t+h, s)-U_{y}(t, s)\right\}\right\|\|f(s, y(s), z(s))\| d s \\
& +\int_{t}^{t+h}\left\|U_{y}(t+h, s)\right\|\|f(s, y(s), z(s))\| d s \\
\|y(t+h)-y(t)\| \leq & r_{1} M h\left\|y_{0}\right\|+r_{1} M h\|g(y)\|+r_{1} M h \int_{0}^{t}\|f(s, y(s), z(s))\| d s \\
& +\int_{t}^{t+h} M\|f(s, y(s), z(s))\| d s \\
\leq & r_{1} M h\left\|y_{0}\right\|+r_{1} M h\|g(y)\| \\
& +r_{1} M h \int_{0}^{t} p(s) \psi(\|y\|+\|z\|) d s+M \int_{0}^{h} p(s+t) \psi(\|y\|+\|z\|) d s \\
\leq & h K_{1} .
\end{aligned}
$$

Thus $\|y(t+h)-y(t)\| \leq h K_{1}$, where $K_{1}$ is constant. Therefore, $y$ is Lipschitz continuous.

The Lipschitz continuity of $y$ combined with the continuity of $f$ imply that $t \rightarrow$ $f(t, y(t), z(t))$ is Lipschitz continuous.

Since $E$ is reflexive and $f$ is Lipschitz continuous, $f$ is differentiable a.e. on $J$ and $f^{\prime} \in L^{1}(J ; E)$.

Hence, $y$ is differentiable a.e. on $J$ and $y^{\prime} \in L^{1}(J ; E)$. Also, $y(t)$ satisfies (1).

Therefore, $y(t)$ is a strong solution of (1) on $J$.

\section{References}

[1] G. Anichini and G. Conti, Boundary value problems with nonlinear boundary conditions, Nonlinearity 1(1988), 1-10.

[2] K. Balachandran and S. Ilamaran, Existence and uniqueness of mild and strong solutions of nonlinear evolution equations with nonlocal conditions, Indian J. Pure Appl. Math. 25 (1994), 411-418.

[3] M. Benchohra and S. K. Ntouyas, Existence of solutions of nonlinear differential equations with nonlocal conditions, J. Math. Anal. Appl. 252(2000), 477-483.

[4] L. Byszweski, Theorems about the existence and uniqueness of solutions of a semilinear evolution nonlocal Cauchy problem, J. Math. Anal. Appl. 162(1991), 494-505.

[5] R. Conti, Recent trends in the theory of boundary value problems for ordinary differential equations, Boll. Un. Mat. Ital. 22(1967), 135-178.

[6] J. K. Hale, Ordinary Differential Equations, Interscience, New York, 1969.

[7] — Evolution equations with nonlocal conditions, J. Math. Anal. Appl. 210(1997), 679-687.

[8] H. Schaefer, Uber die methode der a priori Schranken, Math. Ann. 129(1955), 415-416.

P.S.G. College of Arts Science, Coimbatore, TN-641014, India. 